\title{
Unusual Cause for Pediatric Chest Pain: MDCT Evaluation of Single Coronary Artery
}

\section{Gangadhar K}

Department of Abdominal and intervention radiology, UT Health science center, San antonio, TX, USA

\begin{abstract}
Single coronary artery (SCA) is a rare congenital anomaly in which the entire coronary system arises from a solitary ostium. Anomalies of the coronary arteries are rare conditions that are often asymptomatic. However, if perfusion by the coronary artery is impaired, this anomaly can lead to a life-threatening situation, such as myocardial ischemia or infarction, and sudden cardiac death. ${ }^{5} \mathrm{We}$ report a case of single coronary artery in a six year old girl who presented with acute chest pain.
\end{abstract}

Keywords: Chest pain, Pediatric, Single Coronary Artery (SCA).

\section{Case Report}

A six -year-old girl with history of acute, non-radiating precordial moderate chest pain was admitted to our hospital for evaluation. Physical examination was unremarkable for age. After initial evaluation and treatment, she was referred to department of Radiology for Computed Tomography Coronary Angiography. There was mild ST segment elevation in lateral chest leads of the EKG. CT examination showed single coronary artery originated from right sinus of valsalva with a short main trunk giving off RCA and LMCA. Right dominant coronary system was seen. RCA showed normal caliber and

Corrospondence to: Dr Kiran Gangadhar, MD RD, Department of Abdominal and intervention radiology, UT Health science center, San antonio, TX, USA.

E-mail:- kirang.585@googlemail.com course. LMCA after taking origin from common trunk had a complete intraseptal course with myocardial bridging in the distal

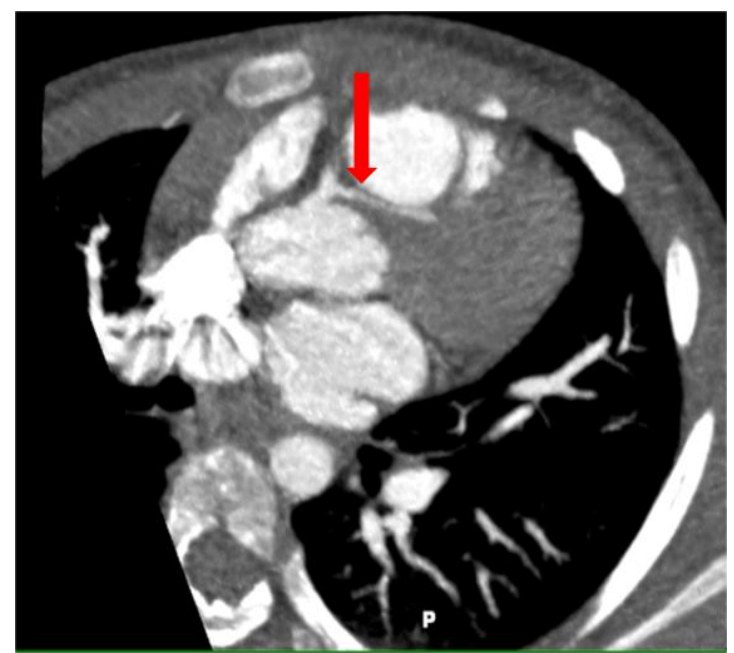

Fig. 1: Axial CT coronary angiography showing Single coronary artery (SCA) originating from right sinus of valsalva with a short main trunk giving off RCA and LMCA(arrow). 
$2 / 3^{\text {rd }}$. An arc like configuration of LMCA was seen coursing along inter ventricular groove giving off small LAD and multiple diagonal branches. After the arc like configuration LCX was seen as direct continuity of the arc coursing along left atrio-ventricular groove. Cardiac chambers and myocardium appeared normal. Great vessels had normal origin and course. These anomalies were reported to the pediatrician and the girl was referred to cardiothoracic vascular surgery for further evaluation and management.

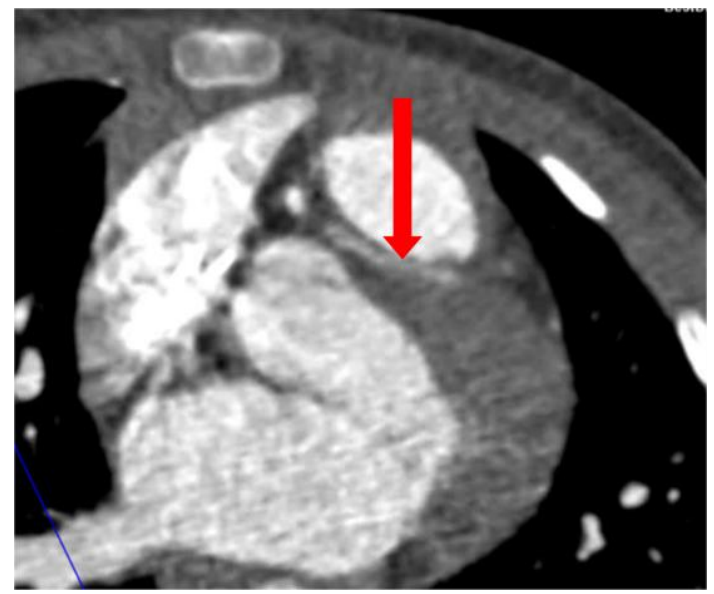

Fig. 2: Axial CT coronary angiography showing LMCA after taking origin from common trunk with complete intraseptal course (arrow) with myocardial bridging in the distal $2 / 3^{\text {rd }}$.

\section{Discussion}

Single coronary artery is a rare congenital anomaly of the coronary arteries where only one coronary artery arises from the aortic trunk by a single coronary ostium, supplying the entire heart. As an isolated finding it occurs in approximately $0.024 \%$ to $0.066 \%$ of the population. In association with certain other congenital anomalies, however, it is found considerably more frequently; the incidence in a series of 54 cases of persistent truncus arteriosus was $18.5 \%$ and in another paper it was reported to be $18 \%^{4}$; in a study of coronary angiograms of 296 patients with Tetralogy of Fallot a single coronary artery was found in seven cases and in pulmonary atresia the incidence is around $17 \% .^{2}$ In 1979, Lipton et al. proposed a very useful angiographic classification'1', further modified in 1990 by Yamanaka and Hobbs.

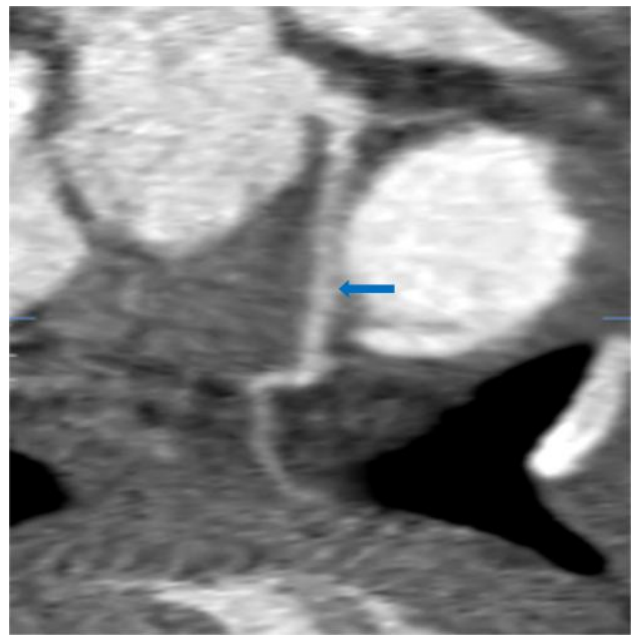

Fig. 3: CT coronary angiography after curved reformatting showing Single coronary artery originating from right sinus valsalva with a short main trunk giving off RCA and LMCA. LMCA after taking origin from common trunk has complete intraseptal course (arrow) with myocardial bridging in the distal $2 / 3^{\text {rd }}$.

In Lipton classification, single coronary artery: According to exit origin ' $R$ ' is the right sinus of valsalva and the ' $L$ ' left sinus of valsalva. According to the anatomical course of the artery, 'I' single coronary artery followed a normal course of the right or the left coronary artery. 'II' single coronary artery, after leaving the right or left coronary sinus to provide the contralateral coronary artery, crosses the base of the heart in a wide range of transverse body. 'III' single coronary artery, after leaving the right coronary sinus of valsalva LAD and LCx arise separately 
from proximal part of the artery. Finally, classified according to relation between anomalous coronary artery with the aorta and pulmonary artery, ' $A$ ' the left main passes anterior to the pulmonary artery, ' $\mathrm{B}$ ' the left main passes between the aorta and pulmonary artery, ' $\mathrm{P}$ ' the left main passes posterior to the aorta. Yamakana et al. have added to this classification the ' $\mathrm{S}$ ' septal (through the interventricular septum) and ' $\mathrm{C}$ ' the combined type.' Our case was type R-II S having complete septal course.

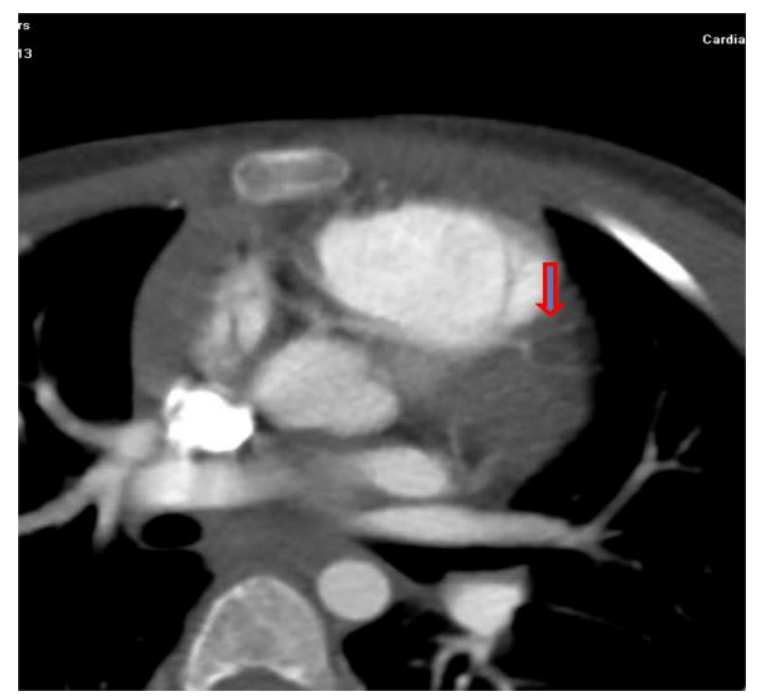

Fig. 4: Axial CT coronary angiography showing Single coronary artery originating from right sinus valsalva with a short main trunk giving off RCA and LMCA. LMCA after taking origin from common trunk has a complete intraseptal course. Arrow pointing at small LAD originating from arc like LMCA with other small diagonal branches.

Previous investigation of coronary anomalies and analysis of the causes of sudden cardiac death has identified the course of the left main coronary artery between the aorta and pulmonary artery as a potential cause of significant coronary ischemia. This anomaly can present with exertional angina, dyspnea, and palpitations, as well as sudden death.
There are several proposed potential mechanisms for the clinical manifestation of this anomaly, such as compression of the left coronary artery between the aorta and pulmonary artery during exercise when the vessels become enlarged; and compromise of the lumen of the left coronary artery due to the acute angle formed at its origin from the right aortic sinus resulting in a slit-like orifice. Other hypothetic mechanisms causing symptoms can be related to a decrease in coronary artery blood flow because of a kinking of the left coronary artery at its origin from the right coronary artery. Kinking may be caused by an increased angulation resulting from distention of the aorta during increased cardiac activity. ${ }^{5}$ The proposed management of this subgroup of patients is surgical because of the high incidence of sudden cardiac death, especially in the young (20 years), who seldom have associated coronary artery disease or other abnormalities. ${ }^{7}$

With the exception of the type RII-B, the main importance of single coronary artery resides in difficulties of diagnosis during coronary angiography and cardiac surgery. Every coronary angiographer should therefore be familiar with the existence and anatomical types of this congenital anomaly. During cardiac surgery unexpected complications may arise. ${ }^{2}$ In a series by $\mathrm{W}$. Desmet et al an anomalous main stem in a patient with type RII-P was accidentally ligated during elective replacement of a bio degenerated artificial valve in the aortic position urging immediate bypass grafting. Twelve other patients underwent successful bypass or valve surgery. One patient died during combined mitral valve and bypass surgery but this death was not related to the presence of a single coronary artery. 


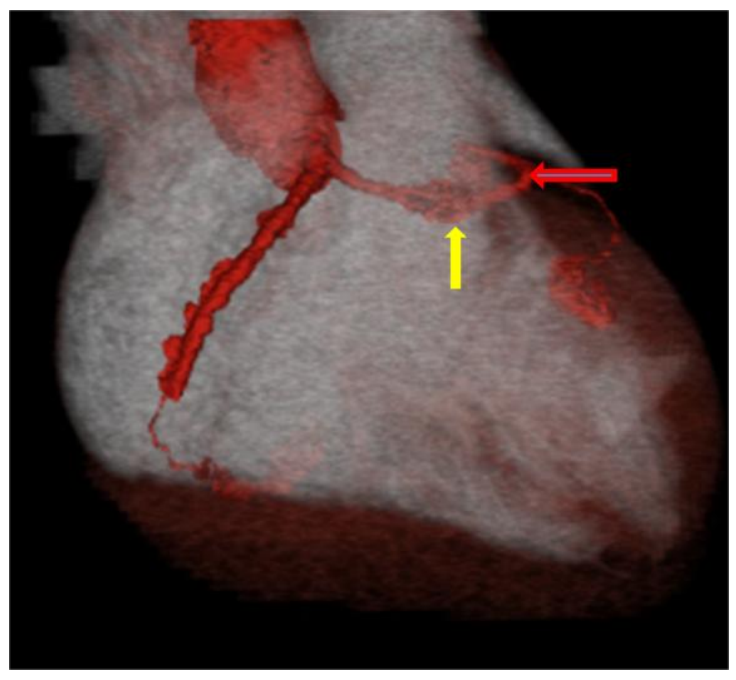

Fig. 5: Volume rendered images with colored reconstruction showing Single coronary artery originated from right sinus of valsalva with a short main trunk giving off RCA and LMCA. RCA showed normal caliber and course. An arc like configuration of LMCA (yellow arrow) was seen coursing along inter ventricular groove. After the arc like configuration LCX (red arrow) was seen as direct continuity of the arc coursing along left atrio-ventricular groove.

The course of anomalous coronary artery and associated with coronary atherosclerotic disease determine treatment strategy. Due to the risk of sudden death in patients whose anomalous coronary artery courses between aorta and pulmonary artery as in our case, coronary artery bypass surgery is useful. Surgical strategy involves the replacement of anomalous coronary artery to coronary sinus appropriately or bypasses surgery. ${ }^{1}$

\section{References}

1. Ozyurtlu F, Acet H, Bilik MZ, Tasal A. A Rare Coronary Artery Anomaly: Single Coronary Artery Originate From Right Sinus Valsalva R-IIP Sub-Group Type. Cardiol Res 2012;3(3):140-142.
2. Desmet W, Vanhaecke J, Vrolix M, Vandewerf F, Piessens J, Willems J, Degeest H. Isolated single coronary artery: a review of 50000consecutive coronary angiographies. European Heart Journal 1992;13:1637-1640.

3. Kaul P, Javangula K. Single left coronary artery with separate origins of proximal and distal right coronary arteries from left anterior descending and circumflex arteries - a previously undescribed coronary circulation. Journal of Cardiothoracic Surgery 2007;2:20.

4. Corbett M, MD, Powers J, MD, King S, MD, Quinn M, MD, Harris D, BA. Single Coronary Artery Journal of the American College of Cardiology Vol. 53, No. 5, 2009.

5. Giorgi B, Dymarkowski S, Rademakers FE, Lebrun F, Bogaert J. Single Coronary Artery as Cause of Acute Myocardial Infarction in a 12-Year-Old Girl: A Comprehensive Approach with MR Imaging. AJR: 179, December 2002.

6. Jahnke C, MD; Nagel E, MD; Ostendorf PC, MD; Tangcharoen T, MD; Fleck E, MD; Paetsch I, MD. Diagnosis of a "Single" Coronary Artery and Determination of Functional Significance of Concomitant Coronary Artery Disease. Circulation 2006;113:e386e387.

7. Horan PG, Murtagh G, McKeown PP. Single coronary artery: a familial clustering. Heart 2003;89:e27. 